\title{
Armes Deutschland
}

\section{Warum wir Perspektiven brauchen}

\author{
Ulrich Schneider
}

\begin{abstract}
Die Armut in Deutschland wächst, der Reichtum auch. Die Perspektivlosigkeit nimmt zu, die Architektur der Sozialversicherungssysteme trägt nicht mehr und der gesellschaftliche Konsens erodiert. Vor diesem Hintergrund ist es Zeit zum Handeln und es gibt auch durchaus vernünftige Optionen dafür.
\end{abstract}

Was war nur los im Frühjahr 2010? Kaum war der Termin durchgesickert, zu dem das Bundesverfassungsgericht sein Regelsatzurteil verkünden wollte, setzte eine in Teilen geradezu hysterisch anmutende Medienberichterstattung ein. (1)

Sollten die Regelsätze infolge des drohenden Richterspruchs erhöht werden, werde sich Arbeit kaum noch lohnen, warnten aufgeregt die einschlägigen $\mathrm{Ga}$ zetten von FAZ bis Bild. Vom Chef der Wirtschaftsweisen, Wolfgang Franz, bis hin zum Forschungsinstitut des Bundes der Steuerzahler reichte der Chor der Warner und Mahner, der da auf die Bühne getrieben wurde. Hektisch drängte es das Kieler Institut für Weltwirtschaft mit einer »Vorabversion « einer eigentlich erst für einen späteren Zeitpunkt vorgesehenen Studie zum Lohnabstand in die Öffentlichkeit. (2)

Die Botschaft war immer die gleiche: Hartz IV macht faul! Oder vornehmer: Eine Erhöhung der Regelsätze nimmt den Arbeitsanreiz. Zum Zwecke der Illustration zerrten Talkshows von Beckmann bis Maischberger einen gewissen Arno Dübel ins Rampenlicht, einen etwas skurrilen Menschen mit einschlägiger RTLDoku-Soap-Karriere, der nach eigenen Angaben so gut wie noch nie gearbeitet

\section{Dr. Ulrich Schneider ist}

Hauptgeschäftsführer des Paritätischen Wohlfahrtsverbandes.

Internet http://www.der-paritaetische.de hatte und dies dank Hartz IV auch nicht müsse. (3) Westerwelles schrille Ausfälle zur »spätrömischen Dekadenz« und zum "anstrengungslosen Wohlstand « fügten sich nahtlos ein in diese in ihrer Unverhohlenheit und Massivität bisher nicht erlebte neoliberale Stimmungsmache.

Dem nachdenklicheren und fachkundigeren Publikum blieb nur Kopfschütteln. Doch ließ die Aufgeregtheit ahnen: Es ging nicht lediglich um 20 der 30 Euro mehr »Stütze«. Wir dürfen nicht übersehen: Hartz IV war nicht einfach die $\mathrm{Zu}$ sammenlegung von Arbeitslosenhilfe und Sozialhilfe. Hartz IV war von zentraler strategischer Bedeutung für die weitere Ausdehnung des Niedriglohnsektors, der über 20 Prozent der abhängig Beschäftigten umfasst. Schon Jahre vor Agenda 2010 und Hartz IV predigten die sogenannten Wirtschaftsweisen die vermeintlich arbeitsmarktpolitisch schädliche Wirkung zu hoher Sozialleistungen. (4)

Die Abschaffung der Arbeitslosenhilfe und die Verkürzung der Bezugsdauer des Arbeitslosengeldes waren Programm. Mit der "Agenda 2010 « sah man sich offensichtlich am Ziel. Die Arbeitslosenhilfe war abgeschafft, der Kreis der Anspruchsberechtigten auf Arbeitslosengeld I deutlich verkleinert und die Regelsätze bei Hartz IV trickreich klein gerechnet. Mit dem Bundesverfassungsgerichtsurteil drohte ein herber Rückschlag auf diesem Weg.

Die Akteure schienen zu spüren: Deutschland befindet sich vor einem Scheideweg. Weil es so tief gespalten ist wie nie zuvor, weil auf sozialpolitisch ausgetretenen Pfaden anscheinend nichts mehr gehen will, weil die Systeme der sozialen Sicherung mehr und mehr versagen (5) und weil der Glaube in die Systeme schwindet, ebenso wie der Glaube in eine Politik des "Weiter so". Deutschland steht vor gesellschaftspolitischen Richtungsentscheidungen. Dabei geht es auch um Vorteile und Privilegien. Deshalb die Aufregung.

\section{So tief gespalten wie noch nie}

14,6 Prozent der Bevölkerung in Deutschland waren im Jahr 2009 armutsgefährdet. (6) Ihr Einkommen lag unter 60 Prozent des Durchschnittseinkommens. Dies sind zwölf Millionen Menschen - ein Höchststand in der Geschichte der Bundesrepublik und des wiedervereinten Deutschlands. Bei nach wie vor über sechs Millionen Hartz IVBeziehern, über eine Million Menschen in der Sozialhilfe und fast 300.000 Kindern in Familien, die wegen ihres Niedrigeinkommens einen Kinderzuschlag erhalten, sind derlei Zahlen durchaus plausibel.

Theoretisch müsste dieses gewaltige Ausmaß an Einkommensarmut nicht einmal erschrecken. Dann nämlich nicht, wenn sich die Quoten seit Jahren im Sturzflug befänden oder aber die Einkommensarmen jedes Vierteljahr andere zwölf Millionen wären. Doch sieht die Realität anders aus (7): Um rund 50 Prozent hat die Armut in den letzten Jahren zugenommen. Der langfristige Trend zeigt nach oben. Zwar ist es in den wirtschaftlich guten Jahren zwischen 2004 und Ende 2008 gelungen, diesen Trend zu stoppen. Doch reichte es nicht, um die Einkommensarmut tatsächlich spürbar abzubauen. (8) Arm und Reich entwickeln sich immer weiter auseinander. Immerhin verfügen acht Prozent der Bevölkerung seit Jahren über das Doppelte und mehr des Durchschnittseinkommens. (9)

In einem Land, das auf eine Vermögenssteuer verzichtet wird und in dem die Erbschaftssteuer faktisch keine Rolle spielt, führen solche Einkommensdiskrepanzen zwangsläufig zu einer noch stärkeren Spreizung der Vermögen. Und so wundert es nicht, dass das Durchschnittsvermögen der Menschen in Deutschland zwischen 2002 und 2007 um erfreuliche zehn Prozent auf 88.000 Euro gestiegen ist, doch 27 Prozent der Bevölkerung gar nichts hatten oder aber sogar verschuldet 
waren. (10) Laut dem Finanzdienstleister Creditrefom war 2010 fast jeder zehnte Erwachsene überschuldet. Das sind 6,5 Millionen Menschen, die ihren Zahlungsverpflichtungen nicht nachkommen können, und zwar ohne irgendeine absehbare Perspektive. (11)

\section{Zunehmende Perspektivlosigkeit}

Zunehmende Perspektivlosigkeit ist es damit auch, die die neue Einkommensarmut kennzeichnet und ihre besondere Brisanz begründet.

Gelang es nach einer Studie des Deutschen Instituts für Wirtschaftsforschung zwischen den Jahren 1996 und 2000 immerhin noch fast der Hälfte, aus der Einkommensunterschicht wieder in die Mittelschicht aufzusteigen, war es zwischen 2002 und 2006 gerade noch ein gutes Drittel. (12) Die Hartz IV-Statistik bestätigt den Trend. 71,1 Prozent der Hartz-IV-Bezieher waren Ende 2009 bereits ein Jahr und länger auf diese Hilfe angewiesen. 54,8 Prozent waren es sogar bereits zwei Jahre und länger. (13) Mit dem »Trampolin « oder dem »Sprungbrett in den Arbeitsmarkt ", als das uns Hartz IV politisch schöngeredet werden sollte, haben diese Zahlen nichts zu tun. (14)

Es ist eine zerstörerische Wirkung, die von dieser Perspektivlosigkeit ausgeht. Auf der individuellen Ebene belastet sie psychisch, macht krank. Kindern nimmt sie Lebensmut und Energie. Gesellschaftlich führt sie zur systematischen Überforderung unserer Sicherungssysteme und schließlich zur Erosion der normativen Grundlagen dieser Arbeitsgesellschaft selbst.

Arbeit ist bekanntermaßen mehr als Gelderwerb. Arbeit strukturiert unseren Alltag, Arbeit prägt unsere Familienstrukturen, Arbeit gibt uns Rollenbilder, Arbeit ist mit Status verbunden und prägt einen Großteil unserer Eitelkeiten. Nach wie vor leben wir in einer Arbeitsgesellschaft mit ausgesprochenem Arbeitsethos. Alternative Orientierungen und Rollenmuster stehen dem Einzelnen nicht ohne weiteres zur Verfügung. Der Fall in die Arbeitslosigkeit bedeutet daher weit mehr als Einkommensverlust. Der Fall in die Arbeitslosigkeit bedeutet geradezu einen Zusammenbruch von zum Teil seit
Jahrzehnten gelebten Routinen und Selbstbildern. (15)

Entscheidend dafür, wie dieser Absturz wahrgenommen wird, ist die Perspektive, die der Einzelne hat. Ob es sich lediglich um eine "Durstrecke« handelt, deren Ende bereits absehbar ist oder ob keinerlei Ausweg in Sicht ist, ist entscheidend für das Erleben und die persönliche Entwicklung, die nicht selten von Existenzängsten geprägt ist und irgendwann dem Gefühl, einfach nicht mehr dazuzugehören.

Gerade Kinder brauchen das Gefühl der Zugehörigkeit und die verheißungsvolle Perspektive für ihre Entwicklung wie die Luft zum Atmen. »Der Mensch kann auf der Welt nicht leben, wenn nichts Erfreuliches vor ihm liegt «, erkannte der russische Pädagoge Anton Semjonowitsch Makarenko bereits in den 1930er Jahren. »Die Freude auf das Morgen ist die wahrhafte Stimulanz im menschlichen Leben. « (16) Über seine Pädagogik folgerte er daraus: »Einen Menschen erziehen, heißt in ihm Perspektiven herausbilden. " "Der morgige Tag muss unbedingt besser erscheinen als der heutige «, wurde zum Leitsatz seines erzieherischen Handelns. (17)

Viele Pädagogen werden es bestätigen: Erst die Orientierung auf die Zukunft und die Freude auf den morgigen Tag schaffen die Energie und die seelische Bewegung, die das Kind zu seiner Entfaltung braucht. Die subjektive Vorwegnahme künftiger Optionen durch das Kind ist ständiger Antrieb und Motor von Entwicklung. Wer Kindern jedoch den Glauben an die Perspektive und die Freude auf das Morgen nimmt, lässt Energien versiegen, raubt dem Kind die Kindheit und nimmt dem Pädagogen jede Chance zur Bildung und Erziehung.

Fraglich ist jedoch, woher Kinder und Jugendliche ihren Glauben an Perspektiven nehmen sollen, wenn sie miterleben, wie ihre älteren Geschwister sich hundertfach erfolglos bewerben, und wie sie zu der Überzeugung gelangen sollen, ihre Zukunft selbst in der Hand zu haben, wenn sie erleben müssen, wie ihre Eltern schon über Jahre erfolglos versuchen, aus Hartz IV herauszukommen, aber immer wieder scheitern? Wie sollen diese Kinder Lebensmut entwickeln, wenn zu Hause Resignation und Frust herrschen?

Wir haben Regionen in Deutschland, in denen jedes dritte Kind von Hartz IV lebt.
Wir können uns heute noch gar keine rechte Vorstellung davon machen, was es für die Entwicklung eines Kindes bedeutet, wenn nicht nur die eigenen Eltern und die Nachbarn jahrelang ohne Arbeit sind, sondern der halbe Stadtteil; wenn Arbeitslosigkeit und Hartz IV der Normalfall sind und die Erwerbstätigkeit die Ausnahme bleibt.

Wir sollten uns vor Augen halten, dass in Deutschland in großer Zahl Kinder heranwachsen, die die Situation, dass morgens alle das Haus verlassen, weil sie zur Arbeit oder zur Schule gehen, am ehesten noch aus dem Fernsehen kennen, aber nicht aus dem persönlichen Umfeld.

\section{Das überforderte (Leistungs-) System}

Es gibt keine Alternative zur Perspektive - nicht für den Einzelnen und im Grunde auch nicht für weite Teile unseres sozialen Sicherungssystems. Mit ihrem Äquivalenzprinzip haben die tragenden Säulen dieses Systems - die Arbeitslosenund Rentenversicherung - keine Antwort auf Perspektivlosigkeit. Auf dauerhafte Ausgrenzung sind sie einfach nicht eingestellt. Schutz vor Armut bieten sie dem, der ohnehin ein gutes Erwerbseinkommen hat und nur kurzzeitig arbeitslos ist. (18) Eine zunehmende Zahl von Mehrfach- und Langzeitarbeitslosen und ein wachsender Niedriglohnsektor lassen dieses System dagegen zunehmend ins Leere laufen.

Als in der Krise Mitte der 1970er Jahre die Arbeitslosenzahl auf über eine Million hochschnellte, erhielten immerhin zwei Drittel von ihnen Arbeitslosengeld. 1990 war es mit 42 Prozent nicht einmal mehr die Hälfte und heute ist es gerade noch ein Drittel der registrierten Arbeitslosen Resultat einer sich zunehmend verhärtenden Arbeitslosigkeit und politisch restriktiver Eingriffe in die Arbeitslosenversicherung selbst. (19)

Und selbst für das verbleibende Drittel gilt: Wer im Niedriglohnsektor von Löhnen leben muss, mit denen er bereits als Erwerbstätiger kaum über Hartz IV liegt, den wird im Falle des Arbeitsplatzverlustes das Arbeitslosengeld I nicht vor der Hartz-IV-Armut schützen.

Ganz ähnlich sieht es bei der gesetzlichen Rentenversicherung aus, nur dass die Auswirkungen von Langzeitarbeitslo- 
sigkeit, prekären Beschäftigungsverhältnissen und Niedriglohnsektor hier naturgemäß erst mit zeitlichem Abstand durchschlagen.

Die ausgesprochen niedrige Quote von 2,4 Prozent der älteren Menschen, die derzeit auf Grundsicherung angewiesen sind, darf nicht darüber hinwegtäuschen, dass eine rapide Zunahme der Altersarmut in den nächsten 15 bis 20 Jahren fast schon unausweichlich ist, wenn nicht massiv gegengesteuert wird. Je mehr ehemals Langzeit- und Mehrfacharbeitslose und Niedriglohnbezieher oder prekär Beschäftige das Rentenalter erreichen, umso schärfer stellt sich erneut das Problem der Altersarmut und umso deutlicher wird der armutspolitische Funktionsverlust der gesetzlichen Rentenversicherung.

Die tradierten Prinzipien des bundesdeutschen Sozialstaates sind in jeder Hinsicht an ihre Grenzen gestoßen. Nicht nur, dass sie in immer weniger Fällen vor Armut schützen. Die Legitimation der Arbeitslosen- und Rentenversicherung selbst steht auf dem Spiel. Insbesondere für Menschen im Niedriglohnsektor gilt, dass ihnen Beiträge abverlangt werden, wohl wissend, dass sie weder bei Arbeitslosigkeit noch im Alter durch diese Kassen vor Armut geschützt werden. Da Möglichkeiten der privaten Vorsorge in aller Regel ebenfalls nicht gegeben sind, werden sie zu ihrem Arbeitslosengeld oder ihrer kleinen Rente zusätzlich auf Hartz IV und Altersgrundsicherung beantragen müssen. Damit jedoch verfügen sie über das gleiche Einkommen, als wenn sie niemals in die Kassen eingezahlt hätten.

Schließlich gefährdet die Perspektivlosigkeit den notwendigen ideellen Grundkonsens dieser Arbeitsgesellschaft. Es ist die Leistungsnorm, die ihr ideelles Fundament bildet. Wer etwas leistet, verdient Belohnung. Wer guten (Leistungs-)willens ist, bekommt sogar sozialen Schutz; Jeder nach seiner Leistung, jeder nach seinen (Versicherungs-)beiträgen. (20) Die Solidargemeinschaft ist eine Gemeinschaft von Leistungserbringern. Wer hingegen nichts leistet, schließt sich aus. Unverhohlen wertend sprechen wir von Leistungswilligen, Leistungsträgern oder Leistungsverweigerern.

Im Grunde belügen wir uns mit dem Leistungsbegriff jedoch gleich doppelt. Sagen wir Leistung, meinen wir eigentlich Erfolg. Und sagen wir Erfolg, meinen wir in Wirklichkeit Geld. Mit dieser Ver- wechslung wird der ohnehin schon kuriose Wert Leistungsgerechtigkeit endgültig zur Ideologie im schlechten Sinne, zur Werte-Lüge im Interesse von Vorteilsnehmern.

War das Leistungsideal damit als normatives Grundkonzept der bundesrepublikanischen Gesellschaft immer schon fragil, so trug es doch. Selbst diejenigen schienen sich an die ideologische Wertelüge von der Leistungsgerechtigkeit gewöhnt zu haben, die sich ganz unten auf der Leistungs-, Erfolgs- oder Einkommenstreppe befanden. Bescheidenheit war schon immer ihre Tugend, das Motto »Geld allein macht nicht glücklich « ihr Trost, die Ziehung der Lottozahlen ihre Hoffnung. Die Empörung über die tatsächlichen Einkommensungerechtigkeiten hat sich viele Jahre lang allenfalls im Stammtisch-Geschimpfe über »die da oben« Luft gemacht, womit »die da oben « ganz gut leben konnten.

Dass das Leistungsgerechtigkeitsideal gesellschaftlich tragen konnte, dürfe wesentlich damit zusammenhängen, dass sein Grundversprechen im Großen und Ganzen noch halbwegs funktionierte: Jeder, der guten Willens ist und sich anstrengt, hat eine Perspektive. Spätestens jedoch, wenn Menschen in großer Zahl zwar Leistungen erbringen wollen, aber nicht die Chance dazu erhalten, verliert ein soziales Gerechtigkeitsideal, das sich aus dem Leistungsprinzip speist, seine Legitimation, seine Akzeptanz und damit letztlich seine Fähigkeit, gesellschaftlichen Konsens zu erzeugen. Wenn Millionen von Menschen keine befriedigende Perspektive mehr sehen und wenn Millionen von Kindern nicht erlernen, an Perspektiven glauben zu können, verliert das Leistungsideal seine integrierende Kraft. Menschen und unter ihnen vor allem die Kinder und Jugendlichen ohne Chancen brechen aus, suchen sich ihre eigenen Normen, schaffen sich ihre eigenen Welten und ihre eigenen Prinzipien. Es ist eine eigene Ordnung, ein eigenes Werte- und Beziehungsgeflecht, das es ihnen wieder erlaubt subjektiv erfolgreich zu sein. Die Gesellschaft zerfällt.

\section{Was wir tun müssen}

Die Einkommensarmut wächst, der Reichtum auch, die Perspektivlosigkeit nimmt zu, die Architektur der Sozialver- sicherungssysteme trägt nicht mehr, der gesellschaftliche Konsens erodiert. Vor diesem Hintergrund sind verschiedene Optionen und Szenarien denkbar.

So könnten wir einfach so weitermachen wie bisher: Niveausenkungen und Leistungskürzungen für diejenigen, die eigentlich am dringendsten auf sozialen Schutz angewiesen sind. Sie bekommen einen Demographiefaktor in der Rentenversicherung, eine verkürzte Bezugsdauer im Arbeitslosengeld oder aber bis zur Lächerlichkeit klein gerechnete Hartz IVRegelsätze. Auf der anderen Seite eine anspruchsvolle Sozialpolitik für die sogenannte Mitte, die sogenannten Leistungsträger dieser Gesellschaft. Sie bekommen Elterngeld, bessere Kinderfreibeträge, einen Zuschuss zur RiesterRente oder Steuervergünstigungen für ihre Haushaltshilfen.

Es ist eine Strategie des sukzessiven Rückzugs des Staates aus seinen sozialstaatlichen Verpflichtungen. (21) Es ist ein staatliches Problemlösungsverhalten, das bereits Ende der 1970er Jahre Einzug in die bundesrepublikanische Sozialpolitik hielt und seitdem immer stärker und unverblümter zum Muster unterschiedlicher familien- und sozialstaatlichen Entscheidungen wurde: die weitere Marginalisierung ohnehin marginalisierter Gruppen, damit für den Rest der Gesellschaft und damit vor allem für den Staat selbst alles beim Alten bleiben kann. 40 Euro mehr Steuerersparnis monatlich pro Kind für den Spitzenverdiener, 20 Euro mehr Kindergeld für den Normalverdiener und den Hartz IV-Beziehern wird das Elterngeld gestrichen. Es ist nur ein Beispiel aus jüngster Zeit für diese opportunistische und damit durchaus mehrheitsfähige Rückzugsstrategie auf Kosten der Ärmsten.

Im Ergebnis der vielen Einzelentscheidungen koppelt sich der Staat mehr und mehr ab von den realen sozialen Problemen in dieser Gesellschaft und ersetzt tatsächliche Problemlösung durch Symbolpolitik, die entschiedenes Handeln suggerieren soll. Wo eine Bildungsoffensive für die ärmsten Kinder angezeigt wäre, eine echte Offensive in der Schulpolitik und der Jugendhilfe, werden Zehn-Euro-Gutscheine angeboten und diese Farce auch noch als Paradigmenwechsel in der Armenpolitik verkauft. Wo eine Pflegeoffensive und eine echte Aufwertung von Pflegeberufen angezeigt wären, werden 


\section{Anmerkungen}

(1) Der Beitrag stellt die Zusammenfassung einiger Thesen aus dem Buch »Armes Deutschland « dar (vgl. Abbildung).

(2) Siehe »Armes Deutschland «, Kapitel »Weichenstellungen «, S. $196 \mathrm{ff}$.

(3) Siehe "Armes Deutschland «, Exkurs »Stille Helden und Arno Dübel «, S. $209 \mathrm{ff}$.

(4) Siehe "Armes Deutschland ", Kapitel »Allzweckwaffe Neoliberalismus Die Leitbilder des Rückzugs «, S. 125 ff.

(5) Siehe »Armes Deutschland «, Kapitel »Unser Sozialstaat an den Grenzen warum Armut hier keinen Platz hat «, S. $52 \mathrm{ff}$.

(6) Siehe Internet http://www.amtlichesozialberichterstattung.de/Tabellen/tabelleA11.html (Internetabfrage 01/2011).

(7) Siehe »Armes Deutschland «, Kapitel »Weniger Arme in Deutschland? Was uns die Statistik verrät und wo sie blufft«, S. $37 \mathrm{ff}$.

(8) Vgl. Frick, Joachim/Grabka, Markus: "Weiterhin hohes Armutsrisiko in Deutschland: Kinder und junge Erwachsene sind besonders betroffen « In: DIW Wochenbericht Nr. 100/2008 S. 2 ff.

(9) Siehe Statistische Ämter des Bundes und der Länder, Internet http://www.amtliche-sozialberichterstattung.de/Tabellen/tabelleA41bund.html (Internetabfrage 01/2010).

(10) Vgl. Frick, Joachim/Grabka, Markus: Gestiegen Vermögensungleichheit in Deutschland. In: DIW Wochenbericht 4/2009 S. 54 ff.

(11) Vgl. Creditreform: Schuldner Atlas Deutschland 2010, S. 4. Internet http://www.creditreform.de/Deutsch/Creditreform/Presse/Archiv/SchuldnerAtlas_Deutschland/2010/Analyse_SchuldnerAtlas_Deutschland_2010.pdf (Abfrage 01/2011).

(12) Vgl. Frick, Joachim/Grabka, Markus 2008: "Schrumpfende Mittelschicht - Anzeichen einer dauerhaften Polarisierung der verfügbaren Einkommen? «In: DIW-Wochenbericht 100/2008.

(13) Vgl. Bundesagentur für Arbeit: Grundsicherung für Arbeitssuchende in Zahlen. November 2010 S. 23.

(14) Siehe »Armes Deutschland «, Kapitel »Das war's dann: Das Scheitern von Hartz IV «, S. 178 ff.

(15) Siehe »Armes Deutschland ", Kapitel »Ausgegrenzt und ohne Perspektive«, S. $168 \mathrm{ff}$.
(16) Siehe Makarenko, Anton Semjonowtsch: Pädagogische Texte. Ein Pädagogisches Poem. Der Weg ins Leben. Paderborn 1976 (erstmals 1933) S. 48.

(17) Makarenko, Anton Semjonowitsch: Pädagogische Werke. Erster Band. Pädagogische Schriften 19221936. Berlin S. 468.

(18) Siehe "Armes Deutschland «, Kapitel »Unser Sozialstaat an den Grenzen: Warum Armut hier keinen Platz hat ", S. $52 \mathrm{ff}$.

(19) Siehe "Armes Deutschland «, Kapitel »Operation gelungen, Patient tot: Die Renten sind unsicher", S. 86 ff.

(20) Siehe "Armes Deutschland ", Kapitel »Das Ende des Prinzips sozialer Gerechtigkeit «, S. $183 \mathrm{ff}$.

(21) Siehe »Armes Deutschland «, Kapitel »Wenn alles zu viel wird: Die Strategie des Rückzugs «, S. 110 ff.

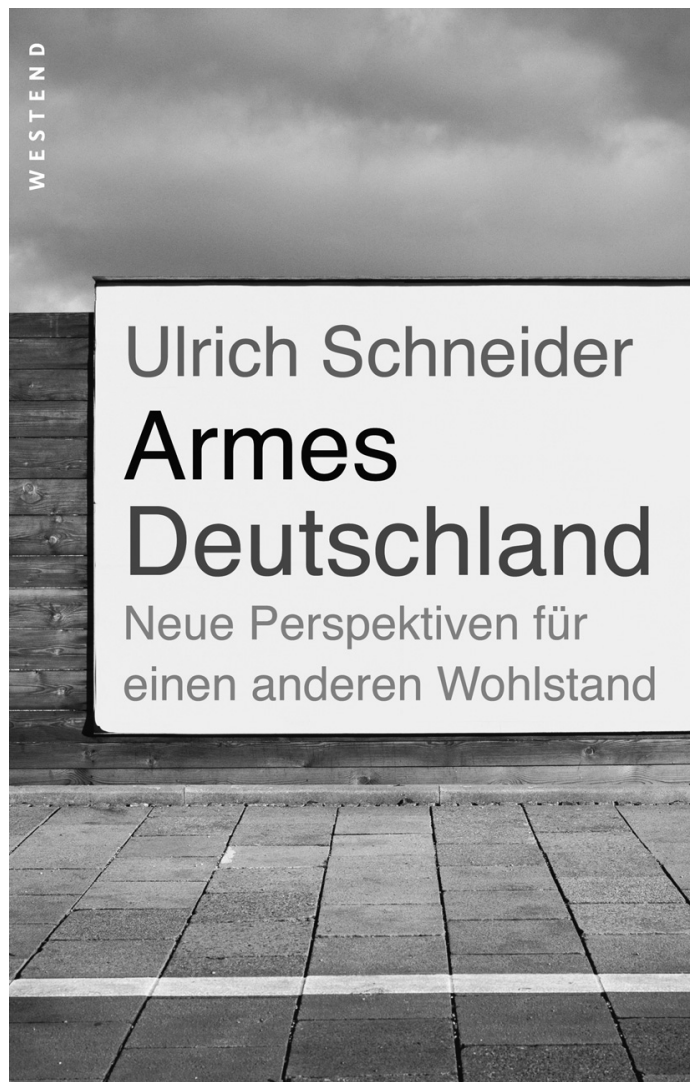

Deutschland steht vor dem Scheideweg. Noch nie lebten so viele Menschen in Armut. Statt die Probleme energisch anzupacken, geht die Politik den kalten Weg der Ausgrenzung. Doch wo Millionen von Menschen ausweglos im Abseits belassen werden, geraten die Fundamente der Bundesrepublik selbst ins Wanken. Ulrich Schneider analysiert die Spaltung der Gesellschaft. Er beschreibt das politische Scheitern und die Strategien und Tricks, mit denen sich die Akteure aus der Verantwortung stehlen. Und er sagt, wie eine andere Politik aussehen könnte und müsste. Es geht um Perspektiven - für jeden einzelnen Menschen und für das Land.

Ulrich Schneider: Armes Deutschland. Neue Perspektiven für einen anderen Wohlstand. Verlag Westend, Frankfurt am Main. 256 Seiten. 16,95 Euro. ISBN 978-3938060-57-5. 
Berichte erstellt, Diskussionen über Transparenz geführt und Imagekampagnen initiiert. Verteilungspolitisch kann so ebenfalls alles beim Alten bleiben - unter Inkaufnahme der langsamen, aber unaufhaltsamen Erosion unseres gesellschaftlichen Zusammenhalts und unserer gesellschaftlicher Grundlagen.

Die Alternative bestünde in Radikalreformen unterschiedlicher Couleur. Schluss mit staatlichen Kassen, staatlichen Versicherungen, Äquivalenzprinzip und Beitragsfinanzierung. Statt dessen Existenzsicherung für die »wirklich Bedürftigen " auf schmalstem Niveau und alles, was darüber hinaus geht, ist Privatsache. Weitgehende Privatisierung ist das, was Marktradikalen ohnehin seit jeher vorschwebt. (22) Mit der Abschaffung der Arbeitslosenhilfe, dem Zurückfahren des Arbeitslosengeldes I und der Einführung der Riesterrente, so ließe sich argumentieren, sind die Weichen ohnehin in diese Richtung gestellt.

Ähnlich radikal bietet sich in der derzeitigen Debatte das bedingungslose Grundeinkommen an: Wo das Leistungsprinzip nicht mehr trägt, müssen halt neue Prinzipien her. Angesichts des zunehmenden Versagens unserer tradierten Systeme und angesichts eines nach wie vor ungebrochenen Bedürfnisses nach sozialer Sicherheit in der deutschen Gesellschaft ist überhaupt nicht erstaunlich, dass Befürworter eines bedingungslosen Grundeinkommens eine immer stärkere Bewegung bilden. (23)

Das bedingungslose Grundeinkommen würde nicht nur eine Radikalreform unserer sozialen Sicherungssysteme darstellen. Seine Protagonisten bieten darüber hinaus ein durchaus neues und positives Menschenbild an, das sich wohltuend abhebt von der bleiernen Misanthropie, welcher Hartz IV zugrunde liegt. Es stellt nichts Geringeres dar als den Versuch, ein gänzlich neues Miteinander in dieser Gesellschaft zu entwickeln, das von Freiheitlichkeit, Vertrauen, vor allem aber von geradezu schrankenlosem Optimismus getragen ist. Dies ist es wohl, was trotz aller realpolitischer Beschränktheiten und offener theoretischer Fragen und Widersprüchlichkeiten seine Attraktivität ausmacht. Es ist wenigstens der Versuch oder Beginn, Sozialpolitik radikal neu und positiv zu denken.

Doch sind derart durchgreifende Systemwechsel, wie immer man zu den ihnen zugrundeliegenden Ideen stehen mag, in absehbarer Zeit aus zwei Gründen ziemlich ausgeschlossen:

- Zum einen gilt zumindest für die Rentenversicherung, dass die erworbenen Rentenansprüche des Beitragszahlers verfassungsrechtlich geschützten Eigentumscharakter haben. Eine Radikalreform müsste daher alle bereits aufgelaufenen Altansprüche bedienen, und gleichzeitig ein neues System aufbauen - ein in der politischen Praxis kaum vorstellbarer Vorgang.

- Zum anderen ist das schon beschriebene Leistungsprinzip, das unserer Sozialversicherung zugrunde liegt, keine bloße technische Größe, sondern schlägt sich ganz offensichtlich direkt in der Mentalität und - trotz seines ideologischen Gehalts - im Gerechtigkeitsempfinden der Versicherten selbst nieder. Wer über noch so kleine Ansprüche an Arbeitslosen- oder Rentenversicherung verfügt, empfindet es in der Regel als zutiefst ungerecht, wenn seine Beitragsleistung in irgendeiner Weise nivelliert oder auch nur relativiert wird - mit anderen Worten: wenn jemand das Gleiche bekommt, obwohl er weniger geleistet und eingezahlt hat.

Eine Reform sozialer Sicherungssysteme kann jedoch immer nur erfolgreich sein, wenn sie die Menschen mit ihrer Mentalität und ihrem Gerechtigkeitsempfinden mitnimmt.

\section{Reform der Sozialversicherung}

Was also praktisch bleibt, ist der mühselige Weg des Reformierens und Weiterentwickelns, um die schleichende Aushöhlung der sozialen Sicherungssysteme zu stoppen. Es gilt, auch Arbeitslosenund Rentenversicherung neue Perspektiven zu geben, anstatt sie dahinsiechen zu lassen. Perspektive bekommen sie dann, wenn sie für den Einzelnen einen Sinn ergeben, und einen Sinn ergeben sie nur dann, wenn sie im Regelfall vor Armut schützen und Beitragszahler besser stellen als Nicht-Beitragszahler.

Für die gesetzliche Rentenversicherung heißt das: Es ist sicherzustellen, dass der Versicherte, der durchschnittlich viele Beitragsjahre beim Rentenzugang aufweist, eine Rente erhält, die über Grundsicherungsniveau liegt. (24) Dazu müssten die unzureichenden Bei- träge am Ende der Erwerbsphase so höher bewertet werden, dass sich dieser Effekt ergibt. Die Differenz wäre aus Steuermitteln $\mathrm{zu}$ finanzieren, da dieser Schritt das rigide Äquivalenzprinzip verlässt und somit der fürsorgerischen Armutspolitik zuzurechnen ist. Für alle, die die notwendigen Beitragsjahre nicht erreicht haben, aber durchaus ihre kleinen Renten oder auch andere kleine Rükkflüsse aus ihrer Altersvorsorge erhalten, wäre ein entsprechender Einkommensfreibetrag in der Altersgrundsicherung einzuräumen. Die vollkommene Anrechnung all dieser Einkommen auf die Altersgrundsicherung wird von den betroffenen Menschen zu Recht als eine Aberkennung ihrer Lebensleistung und ihrer Bemühungen um Altersvorsorge empfunden. Die absehbarer quantitative Zunahme des Problems drängt auf eine rasche Lösung.

Ganz ähnlich verhält es sich mit der Arbeitslosenversicherung. (25) Mit Blick auf den Niedriglohnsektor und einer zunehmenden Zahl von Menschen, bei denen sich Phasen der Erwerbstätigkeit immer wieder abwechseln mit Phasen der Arbeitslosigkeit sind drei Maßnahmen nur naheliegend:

Für ehemals Vollerwerbstätige wäre ein Arbeitslosengeld I auszuzahlen, dessen Höhe auf jeden Fall über Hartz-IV-Niveau liegt. Ein solches Mindestarbeitslosengeld könnte die meisten Haushalte ohne Kinder, die aus dem Niedriglohnsektor heraus arbeitslos werden, vor dem direkten Fall in Hartz schützen. Sind Kinder in diesen Haushalten, sollte ihnen genauso wie erwerbstätigen Familien mit geringem Einkommen der Kinderzuschlag zum Kindergeld gewährt werden, um sie vor Hartz IV zu schützen.

Um Akzeptanz und Funktionsfähigkeit der Arbeitslosenversicherung zu sichern, ist darüber hinaus dafür zu sorgen, dass der Kreis der Anspruchsberechtigten wieder deutlich erweitert wird. Im Zusammenhang mit der Einführung von Hartz IV wurden durch eine rigorose Verkürzung der Bezugszeiten des Arbeitslosengeldes I und eine Reduzierung der sogenannten Rahmenfrist von drei auf zwei Jahre Arbeitslose in großer Zahl aus dem Bezug des Arbeitslosengeldes I ausgesteuert oder gleich ganz ferngehalten. Letzteres meint die Frist, innerhalb derer man zwölf Monate versicherungspflichtige Beschäftigung 
nachweisen muss, um überhaupt Arbeitslosengeld I beantragen zu können. Diese Rahmenfrist wäre schnellstens wieder auf die alten drei Jahre zu erweitern, um dem zunehmenden Phänomen der Mehrfacharbeitslosigkeit gerecht zu werden.

Die Verkürzung der Bezugszeiten wurde mit der offiziellen Begründung vorgenommen, sie diene der Aktivierung der Arbeitslosen. Doch gerade bei dieser bösgläubigen Argumentation stellt sich die Frage, weshalb diejenigen, die sich durch Fortbildung oder andere Maßnahmen unzweifelhaft um ihre Rückkehr in den ersten Arbeitsmarkt bemühen, überhaupt aus dem Arbeitslosengeld-I-Bezug herausfallen sollen. Zumindest für diesen Personenkreis wäre die Bezugsdauer des Arbeitslosengeldes I zu entfristen.

\section{Perspektiven schaffen}

Doch selbst, wenn wir mit solchen Maßnahmen unseren Versicherungen wieder auf die Beine geholfen haben: Wo immer mehr Menschen ohne echte Perspektiven dastehen, ist es mit Geld allein nicht mehr getan. Es müssen Perspektiven her - nicht abstrakt und allgemein, sondern in jedem Einzelfall, ganz konkret. Auf dieses Ziel hin sind unsere bildungsund arbeitsmarktpolitischen Maßnahmen wo immer möglich auszurichten, sollen sie soziale Probleme nicht nur verwalten, sondern lösen. (26)

Doch sind wir derzeit von einer solchen Sozial- und Bildungspolitik weit entfernt. Das "Fordern und Fördern « in Hartz IV entpuppt sich bei näherem Hinsehen als ein nicht selten aktionistisch anmutendes Maßnahmengewusel, das deshalb so stark von Zwang und Sanktionsdruck durchdrungen ist, weil man gerade nicht in der Lage ist, wirkliche Perspektiven zu bieten.

Menschen verrichten Ein-Euro-Jobs, obwohl klar ist, dass dieser Weg nur in Ausnahmefällen in den ersten Arbeitsmarkt führt. Sie werden zum Teil zu Jobs gezwungen, die weit unterhalb ihrer Qualifikation liegen und die sie als weitere Stufe ihres beruflichen Abstiegs empfinden. "Aktivierung " wurde mit Hartz IV versprochen - und mehr ist es wirklich nicht. Die Arbeitssuchenden werden »in Bewegung gehalten «. Mit Perspektiven und Zielgerichtetheit hat das erst einmal nichts $\mathrm{zu}$ tun, wie die einschlägigen Untersuchungen zeigen. Statt auf einem Laufband zum ersten Arbeitsmarkt finden sich zu viele im Hamsterrad wieder. Nicht viel besser sieht es an unseren Schulen aus. So wichtig Bildung und Bildungsabschlüsse in dieser Erwerbsgesellschaft für die Chancen unserer Schüler auch sind - es sind abstrakte Möglichkeiten. Für denjenigen, dem die Schule leicht fällt, der Unterstützung von zu Hause erfährt und für den die Erwerbstätigkeit seiner Eltern etwas ganz Selbstverständliches ist, scheinen sie während der gesamten Schulzeit greifbar und fast selbstverständlich. Wer jedoch keine guten Startvoraussetzungen mitbringt, wer schon aufgrund der Einkommenssituation seiner Eltern ausgegrenzt ist und mit der Erfahrung der schier aussichtslos wirkenden Arbeitssuche seiner Eltern und der genauso deprimierenden Lehrstellensuche seiner älteren Geschwister aufwächst, für den bleiben sie immer abstrakt. Es kann nicht funktionieren, ein Kind oder einen Jugendlichen zu - aus seiner Sicht - sinnlosen Anstrengung motivieren zu wollen. Um Kinder- und Jugendliche aus sozial unterprivilegierten Umfeldern zu motivieren, müssen die Perspektiven konkret und damit glaubhaft sein.

Unsere Sozial- und Bildungspolitik wird nur dann eine Antwort auf die Armutsfrage finden, wenn sie den Armen ganz konkrete Perspektiven bietet und nicht lediglich irgendwelche Bildungsinhalte vermittelt. Bildung mag die notwendige Bedingung sein, die ganz konkrete Perspektive die hinreichende. Wir können nur erahnen, was es für das Klima, die Lernbereitschaft und die Leistungsfähigkeit an unseren Hauptschulen bedeuten würden, wenn wir jedem Schüler und jeder Schülerin einen konkreten Ausbildungsplatz versprechen könnten, wenn die Leistung halbwegs stimmt. Gleiches gilt genauso für all die sogenannten Maßnahmen und sozialpädagogischen Hilfen für junge Menschen in Hartz IV - vom Sprachkurs über Bewerbungstraining bis hin zum Ein-Euro-Job, wären sie tatsächlich auf ein konkretes Ziel hin geplant und organisiert, nämlich auf den Ausbildungsplatz oder Arbeitsplatz auf dem ersten Arbeitsmarkt.

Und da der freie Markt offensichtlich nicht in der Lage ist, die notwendigen Ausbildungsstellen und Arbeitsplätze zu schaffen, muss in übergeordnetem Interesse der Staat tätig werden. Experten gehen 80.000 bis 120.000 fehlenden Lehrstellen aus. Wir können nicht einfach auf die demographische Entwicklung setzen. Ganz im Gegenteil: Mit zunehmend alternder Gesellschaft können wir erst recht auf keinen Jugendlichen verzichten. Wir brauchen deutlich mehr Lehrstellen in den Betrieben und außerdem mehr "ausbildungsbegleitende Hilfen « oder sozialpädagogische Begleitung. Schließlich sind neben der Ausbildung in den Betrieben auch mehr staatlich finanzierte außerbetriebliche Ausbildungsplätze bei entsprechend spezialisierten Ausbildungsbetrieben notwendig: nicht nur für die, die bei der betrieblichen Ausbildung leer ausgegangen sind, sondern gezielt für solche jungen Menschen, die den Anforderungen eines Betriebes - aus welchen Gründen auch immer - noch nicht gewachsen sind. Und da es offensichtlich nicht anders geht, muss über eine Ausbildungsplatzabgabe derjenigen Betriebe nachgedacht werden, die nichts oder zu wenig für die Ausbildung ihres Nachwuchses tun. »Fördern und Fordern « - was für jeden arbeitslosen Jugendlichen gilt, sollte auch für die Betriebe zur Regel werden.

Ohne ein effizientes System der beruflichen Ausbildung, das auch die Jugendlichen mitnimmt, die es etwas schwerer haben, werden wir niemals die nötigen Impulse in den Schulen auslösen können, die nötig sind, um tatsächlich so gut wie alle Jugendlichen $\mathrm{zu}$ einem befriedigenden Lernerfolg zu führen. Auch das Problem einer fehlenden Ausbildungsreife einzelner Jugendlicher, über das seitens der Wirtschaft alljährlich geklagt wird, werden wir nur vom Ende her lösen können: Indem wir nämlich genügend Ausbildungsstellen und ausreichend flankierende Hilfen bereitstellen, die den jungen Menschen signalisieren: »Du hast eine Perspektive, wenn du dich anstrengst! «

Unsere Gesellschaft ist im Begriff, regional und sozial auseinanderzubrechen. Unsere sozialen Sicherungssysteme sind an ihren Grenzen angelangt. Alle bildungs-, sozial- und arbeitsmarktpolitischen Maßnahmen müssen folglich danach befragt werden, ob sie die Menschen vor Einkommensarmut und Ausgrenzung bewahren und ob sie konkrete Perspektiven schaffen. Perspektiven für die Armen, Perspektiven für diese Gesellschaft. 